\title{
BMJ Open MRI-based comparative study of different mild cognitive impairment subtypes: protocol for an observational case-control study
}

\author{
Yang Yu, Weina Zhao, Siou Li, Changhao Yin
}

To cite: Yu Y, Zhao W, Li S, et al. MRI-based comparative study of different mild cognitive impairment subtypes: protocol for an observational case-control study. BMJ Open 2017;7: e013432. doi:10.1136/ bmjopen-2016-013432

- Prepublication history for this paper is available online. To view these files please visit the journal online (http://dx.doi.org/10.1136/ bmjopen-2016-013432)

Received 12 July 2016 Revised 13 December 2016 Accepted 17 January 2017

CrossMark

Department of Neurology, Hongqi Hospital of Mudanjiang Medical Universiy, Mudanjiang, Heilongjiang, China

Correspondence to Dr Changhao Yin; yinchangha07916@sina.com

\section{ABSTRACT}

Introduction: Amnestic mild cognitive impairment (aMCl) and vascular mild cognitive impairment (VaMCl) comprise the 2 main types of mild cognitive impairment (MCl). The first condition generally progresses to Alzheimer's disease, whereas the second is likely to develop into vascular dementia (VD). The brain structure and function of patients with $\mathrm{MCl}$ differ from those of normal elderly individuals. However, whether brain structures or functions differ between these $2 \mathrm{MCl}$ subtypes has not been studied. This study is designed to analyse neuroimages of brain in patients with $\mathrm{VaMCl}$ and $\mathrm{aMCl}$ using multimodality MRI (structural MRI (sMRI), functional MRI and diffusion tensor imaging (DTI)).

Methods and analysis: In this study, 80 participants diagnosed with aMCI, 80 participants diagnosed with VaMCl, and 80 age-matched, gender-matched and education-matched normal controls (NCs) will be recruited to the Hongqi Hospital of Mudanjiang Medical University, Heilongjiang, China. All participants will undergo neuroimaging and neuropsychological evaluations. The primary outcome measures will be (1) microstructural alterations revealed by multimodal MRIs, including SMRI, resting-state functional MRI and DTI; and (2) a neuropsychological evaluation, including the Mini-Mental State Examination (MMSE), Montreal Cognitive Assessment (MoCA), Auditory Verbal Learning Test (AVLT), Memory and Executive Screening (MES), trail making test, Stroop colour naming condition and Clinical Dementia Rating (CDR) scale, to evaluate global cognition, memory function, attention, visuospatial skills, processing speed, executive function and emotion, respectively.

Trial registration number: NCT02706210; Pre-results.

\section{INTRODUCTION}

Owing to the prolongation of the life span, the incidence of chronic diseases has increased. Dementia is a progressive brain disease and is one of the main chronic noncommunicable diseases associated with disability and mortality among elderly

\section{Strengths and limitations of this study}

- Comparison of different subtypes of mild cognitive impairment.

- Use of a combination of multimodal imaging systems.

- Lack of clinicopathological diagnosis.

individuals. Dementia resulting from Alzheimer's disease (AD) has received much public attention. Dementia has many causes in addition to $\mathrm{AD}$. Vascular dementia (VD) is also one of the main types of dementia. Mild cognitive impairment (MCI) constitutes an intermediate stage between normal ageing and dementia. ${ }^{1}$ A widely shared view is that future treatment strategies should focus on the treatment of MCI.

The rate of conversion of the amnestic MCI (aMCI) subtype to AD is $10-15 \%$ annually. Research has focused on the predementia stage of $\mathrm{AD}$, which is likely to be the clinical transition stage of $\mathrm{AD} .^{23}$ In addition vascular MCI (VaMCI) represents the early stage of VD. ${ }^{45}$ The pathogenesis of these two diseases differs. aMCI is a neurodegenerative disease caused by endogenous neuronal factors, including $\tau$ and amyloid pathology, ${ }^{6}$ whereas VaMCI is caused by vascular diseases such as infarcts or profuse white matter (WM) disease. ${ }^{4}$ One study has demonstrated that the structure of the hippocampus is different in patients with aMCI and VaMCI. ${ }^{8}$ However, differences in other parts of the brain have not been detected. Both VaMCI and aMCI are associated with deficits in multiple cognitive domains, but they result in mainly the same symptoms of memory deficits. ${ }^{9}{ }^{10}$ Using neuropsychological tests, a previous study indicated that the mode of memory defects in patients with aMCI and VaMCI differs. ${ }^{10} \mathrm{~A}$ considerable body of literature has concluded that patients with VaMCI generally exhibit 
greater impairment of semantic memory, executive/ attentional functioning, and visual-spatial and perceptual skills, whereas the clinical condition of $\mathrm{AD}$ is characterised by deficits in episodic memory. ${ }^{411}{ }^{12}$ Patients with these conditions may exhibit different performances on specific neuropsychological tests. However, few studies have examined the relationship between these two types of MCI regarding brain structure and cognitive behaviour. Determining the conditions that will result in different types of dementia in patients with cognitive impairment may be helpful for understanding the mechanisms of the two forms of MCI.

According to previous publications, both aMCI and VaMCI are types of a 'disconnection syndrome'. ${ }^{13} 14$ By applying structural MRI (sMRI), resting-state functional MRI (fMRI) and diffusion tensor imaging (DTI) technology, we can comprehensively analyse brain structure and function in patients with aMCI and VaMCI directly and non-invasively. Previous studies have detected atrophy of brain volume and abnormal functional connectivity (FC) in patients with MCI, ${ }^{8}{ }^{15-18}$ along with brain structure impairment in VD that is not consistent with $\mathrm{AD}$, especially in the corpus callosum. ${ }^{19}{ }^{20}$ However, whether these structures differ in various types of MCI has not been determined. Moreover, the changes in brain structure and function are not completely similar. ${ }^{21-23}$ In contrast to using a single technique, the brain structure and function can be analysed in an omnidirectional and multiangular manner using multimodal MRI. The use of multimodal MRI could also consolidate our understanding of how functional networks interact with their structural substrates.

This project seeks to reveal neural circuits involved in the development of network connections and changes that occur in the context of diseases involving cognitive impairment. We seek to further understand the pathogenesis of cognitive impairment and discover the relationship between brain structure, function and cognitive behaviour.

\section{METHODS}

\section{Participants}

A total of 240 right-handed participants (80 patients with aMCI, 80 patients with VaMCI, and 80 normal agematched, sex-matched and education-matched controls) will be recruited from the Neurology Department of Hongqi Hospital of Mudanjiang Medical University, Heilongjiang, China. All participants will undergo a baseline evaluation, including complete sociodemographic and clinical data collection. Patient histories will be collected from informants, typically from their spouses or children. Two experienced neurologists will perform the diagnosis of the participants.

\section{Inclusion criteria}

Criteria for aMCl

The diagnosis of aMCI will be based on the recent international consensus criteria as follows: ${ }^{124}$ (1) subjective cognitive symptoms reported by the informant; (2) objective cognitive impairments that do not meet the Diagnostic and Statistical Manual of Mental Disorders (DSM-V) criteria for dementia; (3) normal or nearnormal performance of general cognitive functioning and no or minimum impairments in daily life activities; (4) abnormal memory function documented by an extensive neuropsychological evaluation, normal general cognitive function and a Clinical Dementia Rating (CDR) scale score $=0.5 ;{ }^{25}$ and (5) neuropsychological testing including a Hachinski ischaemic score (HIS) $\leq 4$ and Montreal Cognitive Assessment score (MoCA, Beijing Version).$^{26}$

\section{Criteria for VaMCl}

The diagnosis of VaMCI will be based on the Diagnostic Criteria for Vascular Cognitive Disorders (VCD) ${ }^{45}$ and DSM-V criteria; and vascular aetiology as follows: cognitive impairment due to subcortical small vessel disease ${ }^{27}$ defined as moderate WM changes (at least one region score $<2$ according to the Wahlund rating scale) or multiple lacunar infarcts $(<2)$ on brain imaging that may or may not be suggested by minor neurological signs. Neuropsychological tests for aMCI, MoCA scoring and HIS determination (HIS $\geq 7$ ) will also be performed.

\section{Exclusion criteria}

Participants with the following conditions will be excluded: (1) psychiatric disease (eg, depression, Hamilton Depression Rating Scale (HAMD) >20, Center for Epidemiologic Studies Depression Scale $>21$ ), systemic disease or other neurological disorders; (2) visual or auditory abnormalities, severe aphasia, or palsy that renders clinical assessments infeasible; (3) any medical or psychological conditions that might affect cognitive functioning, including tranquilisers, antianxiolytics, hypnotics, nootropics and cholinomimetic agents; (4) inability to undergo brain MRI, such as claustrophobia; (5) insufficient Mandarin language abilities to complete the assessment; and (6) marked head motion according to MRI.

The exclusion criteria for VaMCI will also include the following: signs of large vascular disease, such as cortical or corticosubcortical or non-lacunar territorial infarcts and watershed infarcts or haemorrhages.

\section{Neuropsychological evaluations}

All participants will receive a battery of neuropsychological tests to assess general mental status and other cognitive domains. These tests will include the CDR scale, ${ }^{1}{ }^{25}$ Mini-Mental State Examination (MMSE), ${ }^{28}$ MoCA, ${ }^{26}$ Auditory Verbal Learning Test (AVLT), ${ }^{29}$ Memory and Executive Screening (MES), ${ }^{30}$ trail making test (TMT) ${ }^{31}$ Stroop colour naming condition, ${ }^{32}$ CDR and HAMD. ${ }^{33}$ These tests will be used to evaluate global cognition, memory function, attention, visuospatial skills, processing speed, executive function and emotion, respectively. 


\section{Brain MRI protocol}

For each participant, conventional brain T1-weighted image (T1WI) and T2-weighted image (T2WI) data will be obtained to exclude serious brain diseases. For inpatients, the data will be collected at Hongqi Hospital of Mudanjiang Medical University. For outpatients, the T1WI and T2WI data may be acquired from other hospitals. The imaging data used for analysis will be acquired using a $3.0 \mathrm{~T}$ Trio Siemens scanner (Magnetom Tim Trio; Siemens Medical Solutions, Erlangen, Germany) at Hongqi Hospital of Mudanjiang Medical University.

\section{Structural MRI}

High-resolution anatomical images will be acquired using a three-dimensional magnetisation-prepared rapid gradient echo T1-weighted sequence with the following parameters: repetition time $(\mathrm{TR})=1900 \mathrm{~ms}$, echo time $(\mathrm{TE})=2.2 \mathrm{~ms}$, inversion time $(\mathrm{TI})=900 \mathrm{~ms}$, flip angle $(\mathrm{FA})=9^{\circ}$, number of slices $=176$, slice thickness $=1 \mathrm{~mm}$, voxel size $=1 \times 1 \times 1 \mathrm{~mm}^{3}$ and matrix $=256 \times 256$. Brain MRIs will be inspected by an experienced neuroradiologist to determine the presence of gross abnormalities.

\section{Functional MRI}

An echo-planar imaging (EPI) sequence with the following parameters will be used: $\mathrm{TR}=2000 \mathrm{~ms}, \mathrm{TE}=40 \mathrm{~ms}$, $\mathrm{FA}=90^{\circ}$, number of slices $=28$, slice thickness $=4 \mathrm{~mm}$, gap $=1 \mathrm{~mm}$, voxel size $=4 \times 4 \times 4 \mathrm{~mm}^{3}$ and matrix $=64 \times 64$. Participants will be asked to lie quietly in the scanner with their eyes closed during data acquisition. Each scan will last for $478 \mathrm{~s}$.

\section{Diffusion tensor imaging}

An EPI sequence will be used in 32 independent, noncollinear directions with a b-value of $1000 \mathrm{~s} / \mathrm{mm}^{2}$, and one additional image with no diffusion weighting $(b=0)$ will be acquired ( $\mathrm{TR}=11000 \mathrm{~ms}, \mathrm{TE}=98 \mathrm{~ms}, \mathrm{FA}=90^{\circ}$, field of view=256 mm $\times 256 \mathrm{~mm}$, imaging matrix $=128 \times 128$, number of slices $=60$ and slice thickness $=2 \mathrm{~mm}$ ). Three acquisitions will be averaged to increase the signal-to-noise ratio.

\section{MRI data analysis}

sMRI data analysis

Both the cortical reconstruction and morphological feature extraction will be conducted using FreeSurfer software (http://surfer.nmr.mgh.harvard.edu/) with a standard cortical automatic handling protocol. First, a grey matter (GM) template will be created, and the GM will be normalised to the template. The data will be registered to a standard anatomical template, ${ }^{34}$ and the resulting aligned $\mathrm{T} 1$ data set will be segmented and converted to a Montreal Neurological Institute (MNI) space atlas MNI152 $1 \mathrm{~mm}$ brain template using the DARTEL toolbox $^{35}$ and corrected for bias-field inhomogeneity. Subsequently, the images will be skull-stripped using a watershed algorithm ${ }^{36}$ then segmented into subcortical WM and deep GM volumetric structures. ${ }^{37} 38$ The initial tessellation will be conducted for anatomical topological defects. Finally, a variety of morphological features at each vertex on the pial surface will be computed. The parameters will include the entire GM volume, cortical thickness and surface area.

\section{fMRI data analysis}

Image preprocessing will be performed using SPM V.8 (http://www.fil.ion.ucl.ac.uk/spm/) and the Data Processing Assistant for resting-state fMRI. ${ }^{39}$ Preprocessing procedures will be performed, including removal of the first 10 volumes, slice timing and head motion correction. All fMRI data will satisfy the criteria of spatial movement in any direction $<3 \mathrm{~mm}$ or $3^{\circ}$, and the participants will exhibit no significant group differences in the head motion parameters (ie, three translation and three rotation parameters). To spatially normalise the fMRI data, the T1 data used in sMRI will be normalised to the mean functional data, segmented and transformed into MNI152 space using the DARTEL toolbox,,${ }^{35} 40$ and a group template will be generated. Next, the motion corrected functional volumes will be specifically normalised to the group template using the transfer parameter estimated by DARTEL segmentation and resampled to $3 \mathrm{~mm}$ isotropic voxels. Furthermore, the functional images will be spatially smoothed with a $4 \mathrm{~mm}$ Gaussian kernel. Linear detrend and temporal bandpass filtering $(0.01-0.08 \mathrm{~Hz})$ will be performed to reduce the effects of low-frequency drift and high-frequency physiological noise. Finally, several nuisance signals will be regressed from the data, including the six motion parameters and the global, WM, and cerebrospinal fluid signals. FC analysis will be performed using DPARSF software (http:// www.restfmri.net/forum/DPARSF).

To perform the whole-brain resting-state FC (rsFC) analysis, Pearson's correlations between the time courses of all pairs of voxels will be computed, resulting in a whole-brain connectivity matrix for each participant. This procedure will be limited within a GM mask, which is typically generated by a specific thresholding (previous cut-off $=0.2$ ) of the mean map of all GM maps involving all participants without cerebella. These individual correlation matrices will then be transformed as a z-score matrix using Fisher's r-to-z transformation to improve normality. Then, the FC strength (FCS) will be computed as the sum of the connections between a given voxel and all other GM voxels. This computation will be conservatively restricted to connections with a correlation coefficient, which could eliminate the weak correlations possibly arising from noise.

\section{DTI data analysis}

DTI data processing will be performed using software from FMRIB Software Library (FSL, http:// http://www. fmrib.ox.ac.uk $/ \mathrm{fsl})^{41}$. Initially, eddy current corrections will be performed to correct gradient-coil distortions and small head motions using affine registration to a reference image (b0 volume). The brain voxels of DTI data 
will be extracted using the Brain Extraction Tool (BET). The maps of diffusion tensor parameters, including fractional anisotropy (FA), axial diffusivity (DA), radial diffusivity (DR) and mean diffusivity (MD), will be calculated using the DTI-FIT tool, which fits a diffusion tensor model to diffusion-weighted images for each voxel. Voxel-wise statistical analysis of the FA, DA, DR and MD data will be performed for regional differences using tract-based spatial statistics (TBSS), which is a wholebrain voxel-wise analysis method. Then, FSL non-linear image registration algorithm will be used. FA maps of all participants will be aligned into a $1 \times 1 \times 1 \mathrm{~mm}^{3}$ standard MNI 152 space. The target template will be the FMRIB58_FA (http://www.fmrib.ox.ac.uk/fsl/data/ FMRIB58_FA). Then, a mean FA image will be created by averaging the aligned FA maps. The mean FA image will be thinned to build a mean FA skeleton representing the centre of all tracts common to all participants in the study. Each participant's aligned FA data will be projected onto the FA skeleton to obtain their FA skeletons and deformation matrixes. Using the deformation matrixes, skeletonised DA, DR and MD maps will be created by the tbss_non_FA tool. The skeletonised FA, DA, DR and MD map images will be subsequently subjected to statistical analysis.

\section{Statistical analysis}

Repeated measures analysis of variance (ANOVA) will be performed to compare the global differences across the three groups, with follow-up post hoc analyses performed as needed to explain the main effects and interactions.

Multivariate analysis of covariance (ANCOVA) will be conducted with the MCI type as the independent variable, each brain volume as a dependent variable, and age and gender as covariates to analyse differences in brain volume by different types of MCI. In addition, Pearson correlations (two-tailed) will be used to investigate correlations between neuropsychological test scores and the GM volume, cortical thickness and surface area.

A general linear model with multivariate ANOVA and post hoc Bonferroni correction will be performed to compare the FA, MD, DA and DR of each tract among the groups. SPSS (V.20; SPSS, Cary, North Carolina, USA) will be used for this statistical analysis.

A one-way ANCOVA will also be performed to determine the main effect of groups on FCS using age and gender as covariates followed by two-sample t-tests for post hoc analyses. The two-sample t-tests post hoc analyses will be conducted within regions exhibiting significant group effects. All the cluster sizes will be determined by Monte Carlo simulations ${ }^{42}$ using the REST AlphaSim utility. ${ }^{43}$

The two-sample t-tests will be performed on the rsFC maps for each seed using age and gender as covariates. The significance level will be set at $\mathrm{p}<0.05$ with a cluster size of $1350 \mathrm{~mm}^{3}$, corresponding to a corrected $\mathrm{p}<0.05$. The analysis mask will be generated by selecting the voxels that may have a significant positive rsFC in any of the two MCI groups. To investigate the relationship

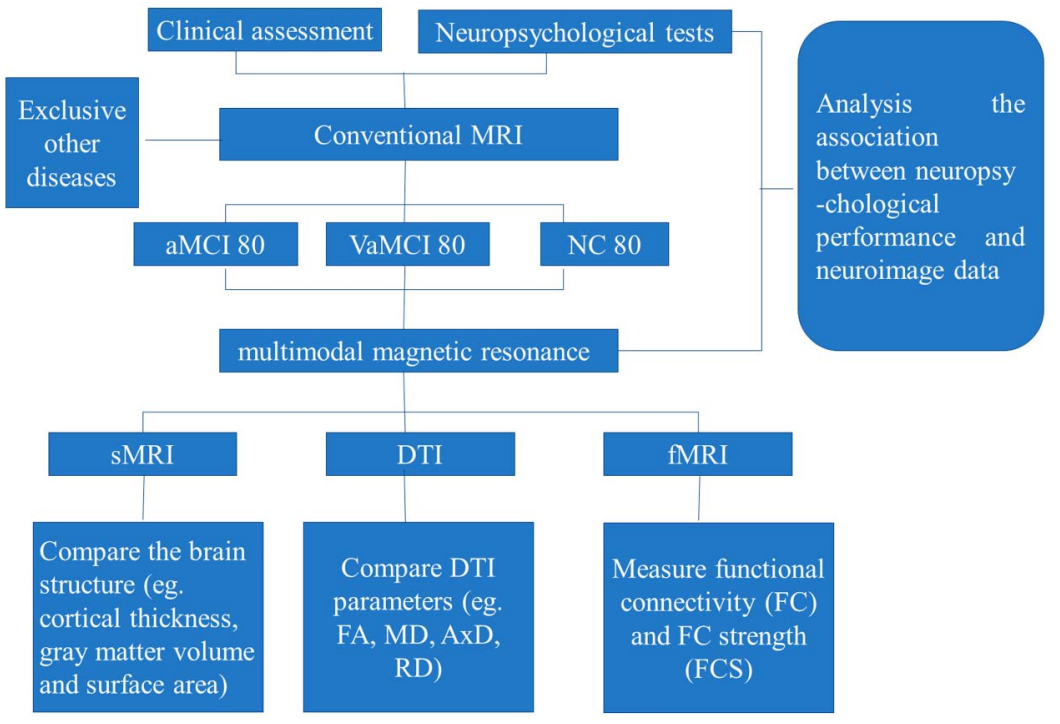

Figure 1 Flow chart of the current prospective diagnostic trial. Eighty participants diagnosed with aMCl, 80 participants diagnosed with $\mathrm{VaMCl}$, and 80 age-matched, gender-matched and education-matched NCs will be subjected to full neuropsychological tests, including the MMSE, MoCA, AVLT, MES, TMT, Stroop colour naming condition, CDR scale and neuroimaging tests, including sMRI, DTI and fMRI. aMCI, amnestic mild cognitive impairment; AVLT, Auditory Verbal Learning Test; CDR, Clinical Dementia Rating; FA, fractional anisotropy; axial diffusivity (AxD); DR, radial diffusivity; DTI, diffusion tensor imaging; fMRI, functional MRI; MD, mean diffusivity; MES, Memory and Executive Screening; MMSE, Mini-Mental State Examination; MoCA, Montreal Cognitive Assessment; NC, normal control; sMRI, structural MRI; TMT, trail making test; VaMCl, vascular mild cognitive impairment. 
among brain function, structure and cognitive behaviour, a general linear model analysis (dependent variable for function: FCS; for structure: FA, MD, DA and $\mathrm{DR}$; independent variables: clinical variables, including MMSE, MoCA, AVLT-immediate recall, AVLT-delayed recall, AVLT-delayed recognition, MES and TMT scores) will be conducted separately.

\section{Association between neuroimaging and neuropsychological performance}

We will analyse the interaction between the parameters acquired by multimodal magnetic resonance and cognition measures.

A scheme of the current prospective trial is described in figure 1.

\section{Ethics and dissemination}

The study will be conducted in accordance with the approved guidelines. Written informed consent will be obtained from all participants, and the participants will be able to withdraw from the study at any time.

\section{DISCUSSION}

In previous studies, researchers have typically focused on the differences between patients and normal elderly individuals, and little attention has been given to the differences between similar diseases. In the late stage of dementia, the WM microstructure in the corpus callosum, especially in the forceps minor, exhibits differences in patients with $\mathrm{VD}$ and $\mathrm{AD} .{ }^{19}$ However, the differences that occur in the early stages of dementia are uncertain. Although the mode of memory defects in patients with aMCI and VaMCI is different, ${ }^{10}$ comparisons of the relationship between these abnormal cognitive behaviours and brain structure and function are lacking. Thus, use of multimodal magnetic resonance to observe neuroimages in the two subtypes of MCI is relevant.

Some limitations of this study should be noted. First, this trial is only an observational study without follow-up; whether these patients will convert to $\mathrm{AD}$ or $\mathrm{VD}$ in the future is uncertain. Therefore, we plan to conduct follow-up to track the dynamic evolution of these patients. Second, our facility is a primary hospital. We do not have a positron emission tomography scanner to increase the precision of diagnosis. However, the diagnosis of the two groups will strictly follow the criteria for the diseases to allow the diagnosis to be as precise as possible. We plan to collect blood and cerebrospinal fluid in future studies.

The purpose for this study is to identify differences in neuroimaging results between patients with aMCI and VaMCI and to explore the possible mechanisms of cognitive disorders in the two diseases. Moreover, we seek to provide scientific evidence for the diagnosis of different subtypes of MCI.

Contributors All authors participated in the design of the study. YY drafted and revised the manuscript. WZ and SL revised the work critically for important intellectual content. CY provided the final approval of the version published. All authors read and approved the final manuscript.

Funding This study is funded by the National Natural Science Foundation of China (grant number 81301188).

Competing interests None declared.

Patient consent Written consent will be obtained from each participant before publishing this study.

Ethics approval Ethical approval for this study has been obtained from the Medical Research Ethics Committee and Institutional Review Board of Hongqi Hospital of Mudanjiang Medical University, Heilongjiang, China.

Provenance and peer review Not commissioned; externally peer reviewed.

Open Access This is an Open Access article distributed in accordance with the Creative Commons Attribution Non Commercial (CC BY-NC 4.0) license, which permits others to distribute, remix, adapt, build upon this work noncommercially, and license their derivative works on different terms, provided the original work is properly cited and the use is non-commercial. See: http:// creativecommons.org/licenses/by-nc/4.0/

\section{REFERENCES}

1. Petersen RC. Mild cognitive impairment as a diagnostic entity. J Intern Med 2004;256:183-94.

2. Hussain $\mathrm{H}$. Conversion from subtypes of mild cognitive impairment to Alzheimer dementia. Neurology 2007;68:409-9.

3. Petersen RC, Doody R, Kurz A, et al. Current concepts in mild cognitive impairment. Arch Neurol 2001;58:1985-92.

4. Sachdev P, Kalaria R, O'Brien J, et al. Diagnostic criteria for vascular cognitive disorders: a VASCOG statement. Alzheimer Dis Assoc Disord 2014;28:206-18.

5. Jin-zhou T, Heng-ge X, Bin Q, et al. Guidelines for the diagnosis of vascular mild cognitive impairment in China. Chin $\mathrm{J}$ Intern Med 2016;55:249-56.

6. Selkoe DJ. Cell biology of protein misfolding: the examples of Alzheimer's and Parkinson's diseases. Nat Cell Biol 2004;6: 1054-61.

7. Gorelick PB, Scuteri A, Black SE, et al. Vascular contributions to cognitive impairment and dementia: a statement for healthcare professionals from the American heart association/American stroke association. Stroke 2011;42:2672-713.

8. $\mathrm{Li} X, \mathrm{Li} \mathrm{D}, \mathrm{Li} \mathrm{Q}$, et al. Hippocampal subfield volumetry in patients with subcortical vascular mild cognitive impairment. Sci Rep 2016;6:20873.

9. Parikh PK, Troyer AK, Maione AM, et al. The impact of memory change on daily life in normal aging and mild cognitive impairment. Gerontologist 2016;56:877-85.

10. Zhou A, Jia J. Different cognitive profiles between mild cognitive impairment due to cerebral small vessel disease and mild cognitive impairment of Alzheimer s disease origin. J Int Neuropsychol Soc 2009;15:898-905.

11. Graham NL, Emery T, Hodges JR. Distinctive cognitive profiles in Alzheimer's disease and subcortical vascular dementia. J Neurol Neurosurg Psychiatr 2004;75:61-71.

12. Tomadesso C, Perrotin A, Mutlu J, et al. Brain structural, functional, and cognitive correlates of recent versus remote autobiographical memories in amnestic mild cognitive impairment. Neuroimage Clin 2015;8:473-82.

13. Nowrangi MA, Lyketsos CG, Leoutsakos JM, et al. Longitudinal, region-specific course of diffusion tensor imaging measures in mild cognitive impairment and Alzheimer's disease. Alzheimers Dement 2013;9:519-28.

14. O'Sullivan M. Diffusion tensor MRI correlates with executive dysfunction in patients with ischaemic leukoaraiosis. J Neurol Neurosurg Psychiatry 2004;75:441-7.

15. Bai F, Xie C, Watson DR, et al. Aberrant hippocampal subregion networks associated with the classifications of aMCl subjects: a longitudinal resting-state study. PLOS ONE 2011;6:e29288.

16. Liu J, Yin C, Xia S, et al. White matter changes in patients with amnestic mild cognitive impairment detected by diffusion tensor imaging. PLOS ONE 2013;8:e59440.

17. Lin $L$, Xue $Y$, Duan $Q$, et al. Microstructural white matter abnormalities and cognitive dysfunction in subcortical ischemic vascular disease: an atlas-based diffusion tensor analysis study. J Mol Neurosci 2015;56:363-70. 
18. Yi L, Wang J, Jia L, et al. Structural and functional changes in subcortical vascular mild cognitive impairment: a combined voxel-based morphometry and resting-state fMRI study. PLOS ONE 2012;7:e44758.

19. Zarei M, Damoiseaux JS, Morgese C, et al. Regional white matter integrity differentiates between vascular dementia and Alzheimer disease. Stroke 2009;40:773-9.

20. Fu JL, Zhang T, Chang C, et al. The value of diffusion tensor imaging in the differential diagnosis of subcortical ischemic vascular dementia and Alzheimer's disease in patients with only mild white matter alterations on T2-weighted images. Acta Radiol 2012;53:312-7.

21. Bai F, Shu N, Yuan Y, et al. Topologically convergent and divergent structural connectivity patterns between patients with remitted geriatric depression and amnestic mild cognitive impairment. J Neurosci 2012;32:4307-18.

22. Zhang J, Wang J, Wu Q, et al. Disrupted brain connectivity networks in drug-naive, first-episode major depressive disorder. Biol Psychiatry 2011;70:334-42.

23. Bullmore E, Sporns O. Complex brain networks: graph theoretical analysis of structural and functional systems. Nat Rev Neurosci 2009;10:186-98.

24. Bowler JV. Modern concept of vascular cognitive impairment. Br Med Bull 2007;83:291-305.

25. Morris JC. The Clinical Dementia Rating (CDR): current version and scoring rules. Neurology 1993;43:2412-14.

26. Lu J, Li D, Li F, et al. Montreal Cognitive Assessment in detecting cognitive impairment in Chinese elderly individuals: a population-based study. J Geriatr Psychiatry Neurol 2012;24:184-90.

27. Erkinjuntti T, Inzitari D, Pantoni L, et al. Research criteria for subcortical vascular dementia in clinical trials. J Neural Transm Suppl 2000;59:23-30.

28. Zhang Z, Hong X, Hui LI. The Mini-Mental State Examination in the Chinese residents population aged 55 years and over in the urban and rural areas of Beijing. Chin $J$ Neurol 1999;03:149-53.
29. Delis DC, Kramer JH, Kaplan E, et al. California verbal learning test: adult version manual. San Antonio, TX, USA: The Psychological Corporation, 1987

30. Guo QH, Zhou B, Zhao QH, et al. Memory and Executive Screening (MES): a brief cognitive test for detecting mild cognitive impairment. BMC Neurol 2012;12:119.

31. Spreen O, Otfried, Strauss E. A compendium of neuropsychological tests. Administration, norms, and commentary (2nd ed). New York, NY: Oxford University Press, 1998.

32. Golden CJ. A manual for the clinical and experimental use of the Stroop color and word test. Stoelting, 1978.

33. Hamilton M. A rating scale for depression. J Neurol Neurosurg Psychiatr 1960;23:56-62.

34. Talairach JJ, Tournoux P. Co-Planar Stereotaxic Atlas of the Human Brain. 3-Dimensional Proportional System: An Approach to Cerebral Imaging. 1988.

35. Ashburner J. A fast diffeomorphic image registration algorithm. Neuroimage 2007;38:95-113.

36. Ségonne F, Dale AM, Busa E, et al. A hybrid approach to the skull stripping problem in MRI. Neuroimage 2004;22:1060-75.

37. Fischl B, Salat DH, Busa E, et al. Whole brain segmentation: automated labeling of neuroanatomical structures in the human brain. Neuron 2002;33:341-55

38. Fischl B, van der Kouwe A, Destrieux C, et al. Automatically parcellating the human cerebral cortex. Cereb Cortex 2004;14:11-22.

39. Chao-Gan Y, Yu-Feng Z. DPARSF: a MATLAB toolbox for "Pipeline" data analysis of resting-state fMRI. Front Syst Neurosci 2010;4:13.

40. Chau W, McIntosh AR. The Talairach coordinate of a point in the MNI space: how to interpret it. Neuroimage 2005;25:408-16.

41. Jenkinson M, Beckmann CF, Behrens TE, et al. FSL. Neuroimage 2012;62:782-90.

42. Ledberg A, Akerman S, Roland PE. Estimation of the probabilities of 3D clusters in functional brain images. Neuroimage 1998;8: 113-28.

43. Song XW, Dong ZY, Long XY, et al. REST: a toolkit for resting-state functional magnetic resonance imaging data processing. PLOS ONE 2011;6:e25031. 